\title{
Passive Control via Slotted Blading in a Compressor Cascade at Stall Condition
}

\author{
M. Ramzi ${ }^{1 \dagger}$ and G. AbdErrahmane ${ }^{2}$ \\ ${ }^{1}$ Mechanical Engineering Department, Faculty of Technology, University of Batna, Algeria \\ ${ }^{2}$ Energy and Turbomachinery Laboratory, University of Tebessa, Algeria \\ †Corresponding Author Email: mdouki_ramzi@yahoo.fr
}

(Received June 9, 2012; accepted August 20, 2012)

\begin{abstract}
This paper was conducted to explore the potential of passive control via slotted bladings in linear cascade configurations under stall condition. Through an extensive 2D-numerical study, the effects of location, width and slope of slots were analyzed and the best configuration was identified. Based on the optimal slot, the 3D aerodynamic performances of cascade were studied and the influence of slotted blading to control endwall flow was investigated. Both 2D and 3D calculations are performed on steady RANS solver with standard k-epsilon turbulence model and low Mach number regime. The total loss coefficient, turning angle and flow visualizations on the blade and end-wall surfaces are adopted to describe the different configurations. The obtained results show, for $2 \mathrm{D}$ situation, that a maximum of almost $28 \%$ reduction in loss coefficient had been reached and the flow turning was increased with approximately $5^{0}$. Concerning 3D flow fields the slots marked their benefit and delays the boundary layer separation on both end wall and blade suction surface at mid span.
\end{abstract}

Keywords: High loading slotted blades, Secondary flow, Separation, Low Mach number.

\section{NOMENCLATURE}

$\mathrm{C}_{\mathrm{f}} \quad$ skin friction coefficient

$\mathrm{C}_{\mathrm{p}} \quad$ static pressure coefficient

c chord length

M Mach number

P static Pressure

$\mathrm{P}_{01}$ inlet stagnation pressure

$\mathrm{R}_{\mathrm{c}}$ coanda radius

$R_{p}$ pressure surface radius

Re Reynolds number

$\mathrm{r}_{\mathrm{L}} \quad$ Slot leading edge radius

$\mathrm{r}_{\mathrm{T}} \quad$ slot trailing edge radius

$\mathrm{S}$ relative dynamic pressure

$\mathrm{t}$ thickness at intersection of slot axis and mean camber line

\section{INTRODUCTION}

It is of main interest to decrease the weight and length of compressor by reducing the number of blades or stages for a desired pressure rise. The problem encountered in front of this reason is the rise in the level of loading and diffusion which the boundary layer separation can be occurred. It is well known that separation is the main factor to reduce drastically the aerodynamic performances of compressors in turboengines. It decreases the operating range by generating instability such as rotating stall and surge, and reduces
$\mathrm{X}$ chordal slot location

$\mathrm{Y}$ slot width

$\mathrm{y}^{+}$non-dimensional distance

$\mathrm{W}_{1}$ inlet velocity

$\alpha_{1}$ angle of attack

$\beta_{1}$ inlet flow angle

$\theta$ turning angle

$\lambda$ stagger angle

$\varpi$ mass averaged total loss coefficient

$\sigma$ solidity

$\psi \quad$ angle formed by slot axis and mean camber line

efficiency by producing high losses. The evident objective of engineers facing this undesirable phenomenon is to control it by mitigating or eliminating the separation zones. A lot of researches have been done in the area of flow control but the passive control method remains the preferable tools because of their simplicity and cost effectiveness. The basic principle of passive flow control is to energize the low momentum layers near solid surface without adding extra energy in order to overcome stronger adverse pressure gradient and therefore avoid the flow separation. All of the 
passive approaches used in turbo machinery such as vortex generators, Gurney flaps, slots and tandem bladings have been derived from methods successfully applied to aircraft wings. The slots represent one of the older methods to control boundary layer. To the author's knowledge, there have been a few previous investigations of slots in real turbo machinery applications. One of the two dimensional investigations which was conducted to determine the potential of slotted cascades for obtaining a wide range of operation and a large stall margin of compressor stages are carried out by Zhou et al. (2008, 2009). In Zhou et al. (2008) work, three kinds of blade slot treatments were designed and the results indicate that the performance of cascade can be improved by effective slot position and structure. In Zhou et al. (2000) work the slot solution was designed on the stator blade in order to explore their effect on the single stage compressor characteristic. The slot effect was positive to improve compressor performance and enlarge stable operation region. As experimentally investigated in annular compressor cascade with slotted blades by Rockenbach et al. (1968, 1970), the effectiveness of the slots appears in the region of mid span, but near both end walls the control gave poor performance and marked their inability to minimize the secondary flow losses. Moreover, the results indicated that no benefit was achieved in the wall region by the addition of wall vortex generators or inter blade secondary flow fences to original slots. Concerning the control with tandem airfoil, the recent three dimensional numerical study which performed by Mc. Glumphy et al. (2010) showed an improvement in the performance over a single blade rotor and the end wall flow losses had an important effect. Yasuo et al. (2005) used a bowed compressor cascade to ameliorate the performances in endwall region. The numerical results indicated that the bowed stacking blade enhanced the spanwise transport of low momentum fluid inside the endwall boundary layer from sidewall towards the midspan. The control with the bowed shape of blade decreased the number of vortices developed in the region of corner stall and reduced the level of total pressure losses. The effects of the control appear remarkably when the blade loading is increased. Quite recently, Hergt et al. (2011) proposed an endwall groove to influence on the secondary flow structure. The numerical and experimental results were promising, the strong interaction of low momentum end wall fluid with the suction side boundary layer was considerably decreased and the secondary flow structure was significantly influenced by the aerodynamic separator. Furthermore, the spanwise as well as pitchwise extension of separation in the corner stall was mitigated and the losses were reduced. The present article focuses on numerical investigation which firstly explores the best configuration of cascade where the slot jet energizes the low momentum flow in order to delay or eliminate the separation boundary layer and thus enhance the aerodynamic performances of the highly loaded compressor cascades. Different slot locations, slot slope angles and slot widths are studied in order to achieve this objective in $2 \mathrm{D}$ configuration. The second part consists in studying the influences of the optimal slot on secondary flow structures at off design condition.

\section{Numerical Procedure}

\subsection{2-D Configuration Cascade}

In the preprocessing step, the geometry and mesh are developed in GAMBIT. The geometry definition gives the study field limited, in streamwise, by inlet and outlet located at approximately 1.2 chords away from the leading edge and trailing edge, respectively, and, in pitchwise, by two periodic identified by solidity $\sigma$ $=1.25$. Between these four boundaries, the high cambered blade NACA $65(18) 10$ profile has been chosen. It is built by creating real edges from a 26 points table, taken from the reference of Emery et al. (1958). The blade is provided by a slot with constant section $Y$ and characterized by a stagger angle $\lambda=13^{0}$ and a chord length $c=0.127 \mathrm{~m}$, Fig. 1 .

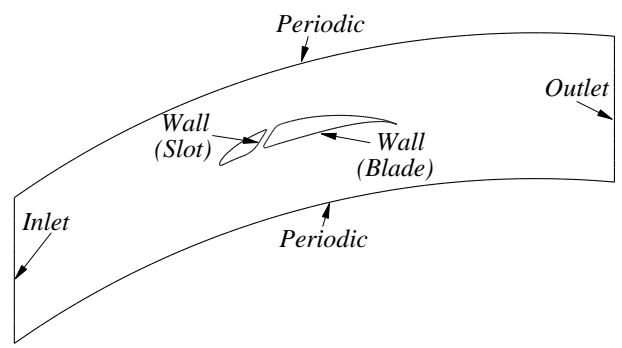

Fig. 1. Geometric model with slotted blading

It is well known that the existence of singular points, which represent in this investigation the slot corners, provoke a pressure gradients between their upstream and downstream and consequently the separation of boundary layer in these zones. The remedy of this problem is to design slot geometry with curves instead broken lines as shown in Fig. 2. The slot data are chosen from the reference reported by Linder $e t$ al. (1966). They are summarized as follow:

\author{
$\mathrm{X}$ : Chordal slot location \\ $Y$ : Width slot \\ $t$ : Thickness at intersection of slot axis and mean \\ camber line \\ $r_{T}$ : Slot trailing edge radius $; 0.000127 \mathrm{~m}$ \\ $r_{L}$ : Slot leading edge radius $; 0.097 t$ \\ $R_{c}$ : Coanda radius $; 0.792 t$ \\ $R_{p}$ : Pressure surface radius; $1.73 t$ \\ $\psi$ : Angle formed by slot axis and mean camber line.
}

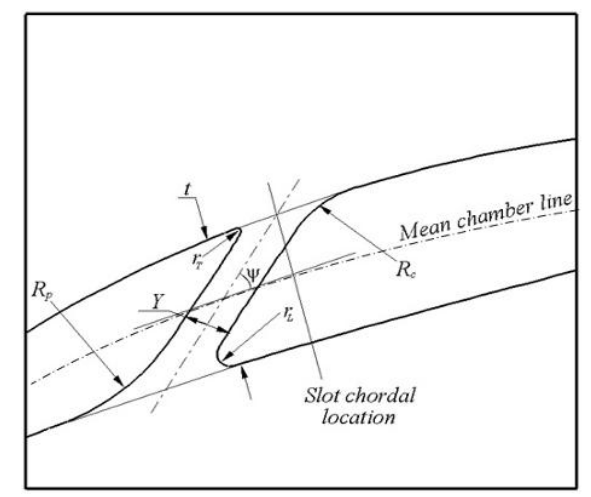

Fig. 2. Slot geometry nomenclature 
The grid generation represents the subdivision of the study field into discrete control volumes. Two types of mesh are applied, a structured mesh in the vicinity of blade surfaces to capture the severe gradients in the region of boundary layer and an unstructured mesh in the remainder of computational domain. The wall functions model needs to adjust the thickness of neighboring cells to blade surface with the value of 0.0005 chords in order to satisfy the condition $30<y^{+}<100$, where, $y^{+}$is the characteristic non dimensional distance from the wall. The total number of cells for a typical cascade configuration is about 26000 . The independence grid-solution is obtained after several attempting improvements. Since each model needs a different mesh, it is inappropriate to show all slotted cascades tested in this work.

Therefore, only representative mesh of slotted cascades with a chordal location $X=0.35 c$, a width $Y=4 \mathrm{~mm}$, and a slope $\psi=45^{\circ}$ is shown in Fig. 3(a). Detailed 2-D mesh configurations are shown in Fig. 3(b) to Fig. 3(d), respectively for the leading edge zone, trailing edge one and in the slot.

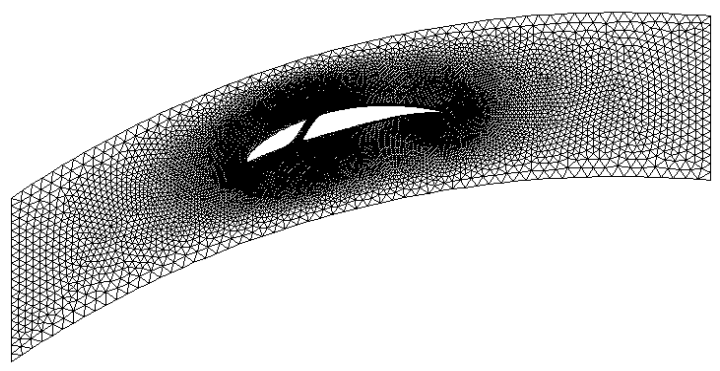

Fig. 3(a). Computational grid for the slotted cascade with $X=0.35 \mathrm{c}, Y=4 \mathrm{~mm}$, and $\psi=45^{\circ}$

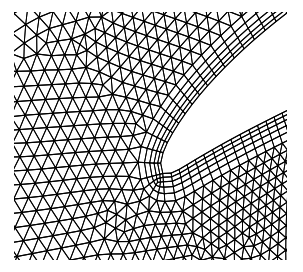

Fig. 3(b). L.E local mesh

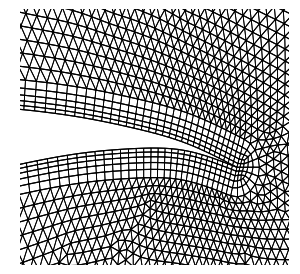

Fig. 3(c). T.E local mesh

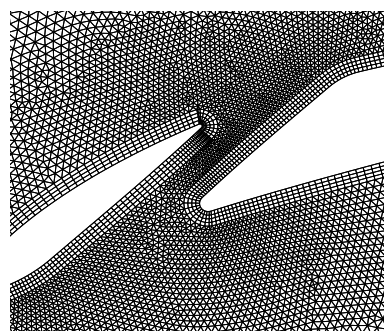

Fig. 3(d). Local mesh around slot location

In the processing step, the FLUENT solver is used as a CFD-tool for solving the governing equations. The flow model considered in the present investigation is based on two dimensional situation, steady state, incompressible regime and $k$ - $\varepsilon$ turbulent model with wall functions. Therefore, the governing equations representing continuity, momentum, $k-\varepsilon$ transport equation are discretized using the finite volume approach applied in FLUENT solver. It is convenient to simulate one flow passage limited by two interfaces because the row compressor represents a cascade with an infinite number of blades. At these interfaces or periodic boundaries the principle of ghost cells is introduced. Consequently, the real and ghost cells are allowed to overlap without need to interpolate the flow variables with another blade passages. At all solid walls such as pressure, suction surfaces, and slot walls; the no slip and impermeability condition is imposed. At the inlet, the velocity components, turbulence intensity and hydraulic diameter are specified. On the contrary, at the outlet, the velocity components and turbulence parameters are extrapolated from neighboring interior cells.

To validate the numerical model; a comparison between computational and experimental results is carried out. The experimental data comes from the Emery et al. (1958) report. The comparison is shown in Fig. 4 for the surface pressure distribution on a NACA 65(18)10 cascade without slots and reported in terms of relative dynamic pressure, $S=2\left(P_{0 I}-P\right) /\left(\rho W_{l}^{2}\right)$, where, $P_{0 I}$ is the upstream stagnation pressure. The operating conditions are set at free-stream Mach number $M=0.085$ and Reynolds number, based on blade chord, $R e=245000$. The cascade has a stagger angle $\lambda=13^{\circ}$, blade angle of attack $\alpha_{l}=17^{0}$ and solidity $\sigma=1.25$. The shown result in Figure 4 and other numerical outputs for many tested configurations give a good agreement with experimental data cited in Emery et al. (1958).

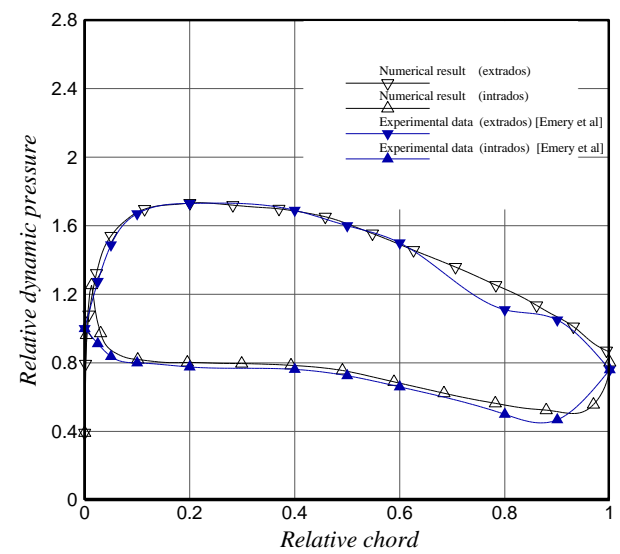

Fig. 4. Comparison between experimental and numerical results for blade surface pressure distributions without slot $\left(\alpha_{I}=17^{\circ}, \lambda=13^{0}\right.$ and $\left.\sigma=1.25\right)$

\subsection{3-D Cascade Configuration}

Before The 3-D geometry is provided by the optimal 2D slot and has been developed with the same precedent cascade parameters: chord length $c=0.127 \mathrm{~m}$, stagger angle $\lambda=13^{\circ}$, solidity $\sigma=1.25$. The aspect ratio $A R=h / c$ which gives the third dimension is equal to unity, Fig. 5 . 


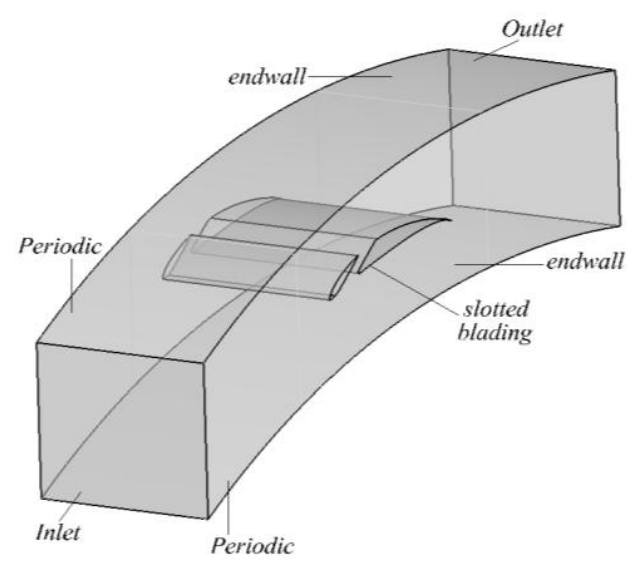

Fig. 5. 3-D domain with boundary conditions for the slotted cascade

The grid generation represents two types of elements, a structured mesh in the regions of inlet and outlet to minimize the number of cells and an unstructured mesh in the blade passage. The endwall region of the cascade was meshed using quadrilateral and triangular elements with refined grid near solid surfaces. The total number of cells for 3-D cascade configuration is about one million, Fig. 6.

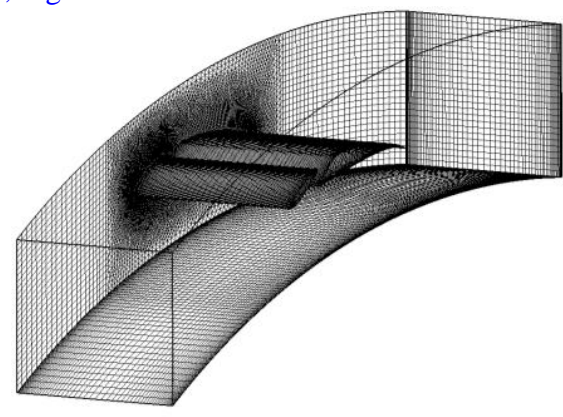

Fig. 6. 3-D representation of computational grid for the slotted cascade

At all solid walls such airfoil, slot and end walls; the noslip and impermeability condition is imposed. At the inlet plane, the velocity distribution and flow angle of the free stream are prescribed. The velocity components and turbulence parameters, at exit plane, are extrapolated from neighboring interior cells. It was assumed that the turbulence intensity of the inlet flow is $1 \%$.

This solver already gave accurate results, comparing Yasuo et al. (2005) experimental results of 3-D flows characteristics for a two dimensional cascade, refering to a NACA65 blading with a camber angle of $30.1^{\circ}$, a stagger angle of $32.2^{\circ}$, a solidity of 1.08 and an aspect ratio of 2.3. The incoming fluid inlet angle was $47.1^{\circ}$ and the Reynolds number based on chord length was 2.2 $10^{5}$.

For this test case geometry, the spanwise distribution of total pressure loss coefficient and deflection angle are shown in Figs. 7(a) and 7(b) respectively. The deflection angle is defined as $\theta=\beta_{1}-\beta_{2}$; where $\beta_{1}$ and $\beta_{2}$ are the angles between inlet and outlet flow, respectively, and the axial direction. Compared to the experimental results, the numerical ones obtained by the present authors are better compared to Yasuo et al. (2005) ones.

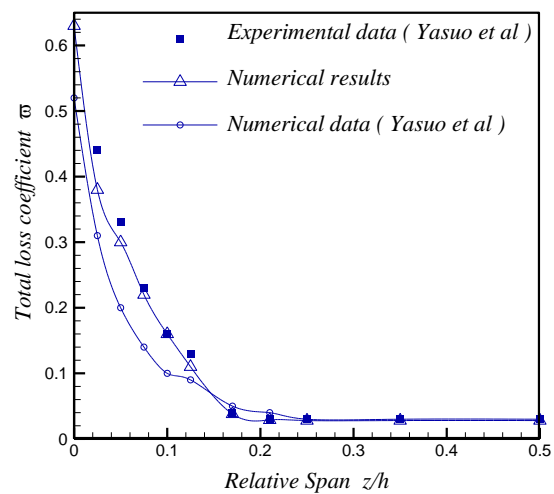

Fig. 7(a). Spanwise distribution of pressure loss coefficient: comparison between experimental and numerical results

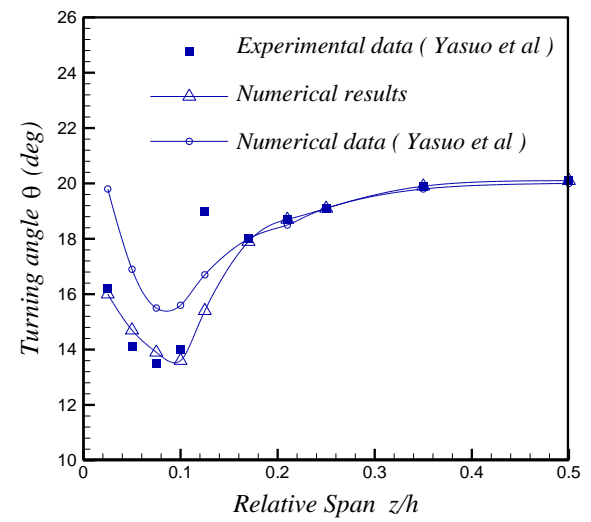

Fig. 7(b). Spanwise distribution of turning angle: comparison between experimental and numerical results

\section{RESULTS AND DISCUSSION}

\subsection{Investigation and Parametric Analysis}

All the parametric study will be carried out on the basis of a cascade configuration with a constant angle of attack $\alpha_{1}=39^{\circ}$ which gives a beginning of separation at about $70 \%$ of the relative chord length. Figure 10 shows this separation zone for which the pressure gradient starts leveling off.

The influences of location, width and slope of the slot are successively examined. Each control parameter is studied independently and the efficiency of control is analyzed on the basis of the mass averaged total loss coefficient and the turning angle.

The objective of this section is to identify the optimal slot location by fixing the width and slope as $Y=2 \mathrm{~mm}$ and $\psi=45^{\circ}$. The analysis is carried out on eight locations, seven adjacent positions are separated with $10 \%$ of the relative chord length and one other position is located at $35 \%$ of the relative chord length. The procedure to determine the location of the slot is to draw the slot centerline at an angle $\psi$ with the mean line and passed it through the suction surface. The 
produced intersection represents the desired chordal slot location $X$ on the suction side. Figure 8 shows that the lower loss coefficients are located in the range of locations between $30 \%$ and $50 \%$ of the relative chord length and the best loss coefficient is identified when the slot is positioned at $35 \%$ of the relative chord length.

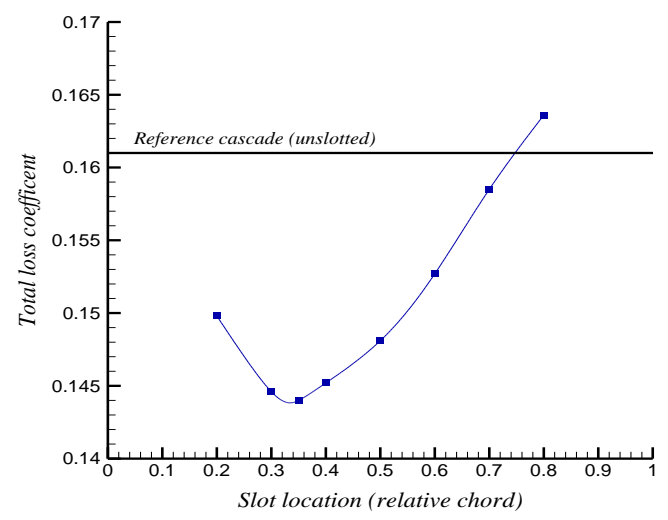

Fig. 8. Loss coefficient for different slot locations $\left(Y=2 \mathrm{~mm}\right.$ and $\left.\psi=45^{\circ}\right)$

First, since the position of separation point is located at about $70 \%$ of the relative chord length and the minimum pressure point is close to the leading edge, Fig. 10, the optimal location $X=0.35 c$ confirms Linder's criterion, which said that the slot would be located approximately halfway between the minimum pressure point and the separation point.

Second, the reason for the higher losses marked in the region downstream the location $X=0.5 c$, in particular in the separation zone, is the insufficient slot jet momentum to energize the surface suction boundary layer. This insufficient slot jet momentum is resulted from the weak difference pressure level between suction and pressure surface shown by the static pressure coefficient distribution in Fig. 10. Concerning the slot located in the detachment zone, it is inadvisable to exhaust the slot flow into the separated region because it cannot effectively turn the primary flow back toward the suction surface.

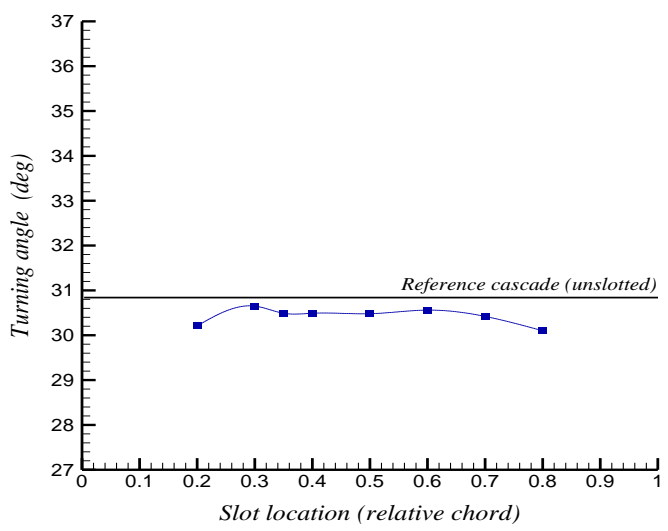

Fig. 9. Turning angle for different slot locations $\left(Y=2 \mathrm{~mm}\right.$ and $\left.\psi=45^{\circ}\right)$

Third, in spite the high difference pressure level in the region upstream the location $X=0.3 c$, illustrated by the static pressure coefficient distribution, Fig. 10, the loss coefficient appear high. This increase is produced by the mixing losses due to the high velocity of the main flow in this region. Figure 9 shows that the different locations of the slot used with the width $Y=2 \mathrm{~mm}$ and the slope $\psi=45^{0}$ influences negatively but slightly the turning angle.

The influence of slot width is studied for the optimal position $X=0.35 \mathrm{c}$ and the fixed value of slot slope $\psi$ $=45^{0}$.

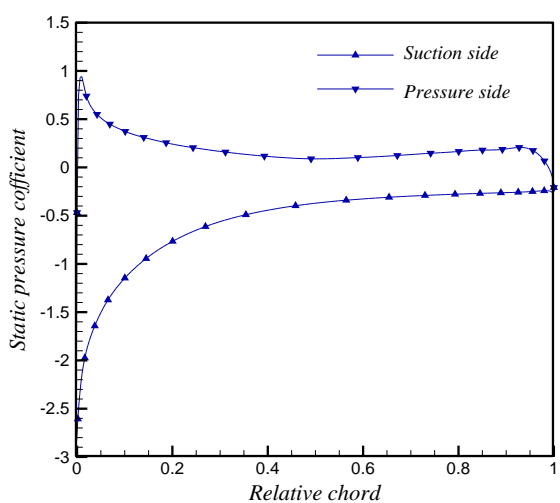

Fig. 10. Static pressure coefficient on blade surfaces without slot $\left(\alpha_{1}=39^{\circ}, \lambda=13^{\circ}\right.$ and $\left.\sigma=1.25\right)$

The results are reported in Fig. 11 and Fig. 12 for the different widths from 1 to $11 \mathrm{~mm}$. The positive effect is obtained when the width is increased as far as the value $Y=6 \mathrm{~mm}$. The two widths $Y=6 \mathrm{~mm}$ and $Y=7 \mathrm{~mm}$ give the same lower value of loss coefficient but the slot width $Y=7 \mathrm{~mm}$ leads to high turning angle, Fig. 12. Therefore, the optimum corresponds to the threshold value $Y=7 \mathrm{~mm}$. Beyond this optimal value, the increasing in the level of losses represents the sign of the thicker of boundary layer set by the higher values of slot width.

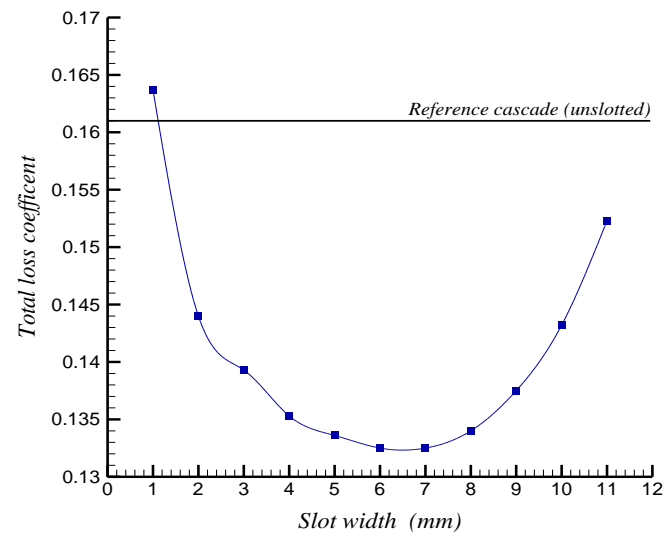

Fig. 11. Loss coefficient for different slot widths $\left(X=0.35 c\right.$ and $\left.\psi=45^{\circ}\right)$

Similarly, in order to analyze the influence of slot slope, the optimal control parameters such the position $X=0.35 \mathrm{c}$ and the width $Y=7 \mathrm{~mm}$ are used for three values of the angle $\psi ; 30^{\circ}, 45^{\circ}$ and $60^{\circ}$. The results, shown in Fig. 13, indicate that the best result corresponds to the lowest tested angle $\psi=30^{\circ}$. In fact, with the fixed exit slot on the position $X=0.35 c$, at suction side, and the different entries slot, at pressure side, which correspond to the angles $\psi=30^{\circ}, 45^{\circ}$ and $60^{\circ}$, the pressure 
difference, between the entry and the exit of slot, increases when the slot slope becomes less stiff. This is confirmed by the static pressure coefficient distribution in Fig. 10. The numerical experimentation presented here provides us different solutions to reduce the mass averaged loss coefficient and increase the turning angle. The best solution is obtained for a slot located at $35 \%$ of relative chord length, a slot width $Y=7 \mathrm{~mm}$ and a slope, defined by the angle between the slot centerline and the mean chamber line, $\psi=30^{\circ}$. The relative reduction of loss coefficient is up to $28.3 \%$ and the turning angle increase with $5^{0}$, Fig. 14.

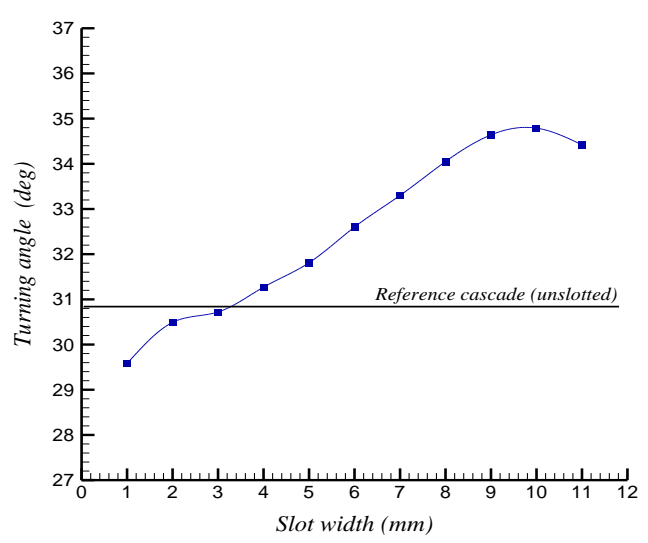

Fig. 12. Turning angle for different slot widths $\left(X=0.35 c\right.$ and $\left.\psi=45^{\circ}\right)$

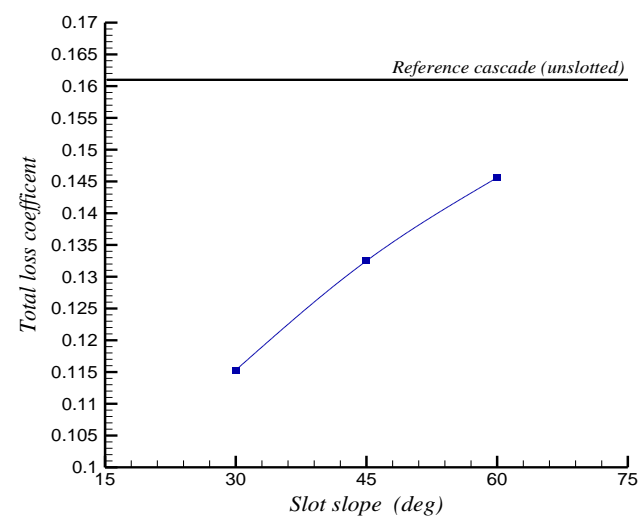

Fig. 13. Loss coefficient for different slot slopes $(X=0.35 c$ and $Y=7 \mathrm{~mm})$

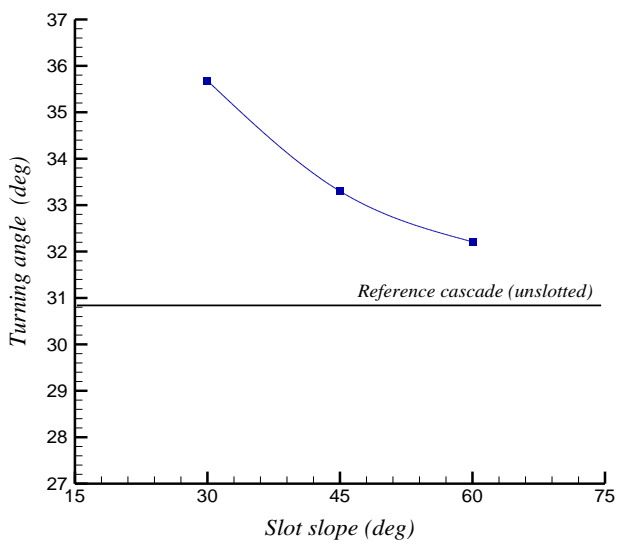

Fig. 14. Turning angle for different slot slopes $(\mathrm{X}=0.35 \mathrm{c}$ and $\mathrm{Y}=7 \mathrm{~mm})$
Figure 15 shows the comparison of velocity magnitude fields and streamlines between the slotted and unslotted cascades and illustrates the significant influence of the optimal slot jet momentum to eliminate boundary layer separation. In this configuration cascade, the passive control with slotted blading proves his efficiency to eliminate the flow detachment.

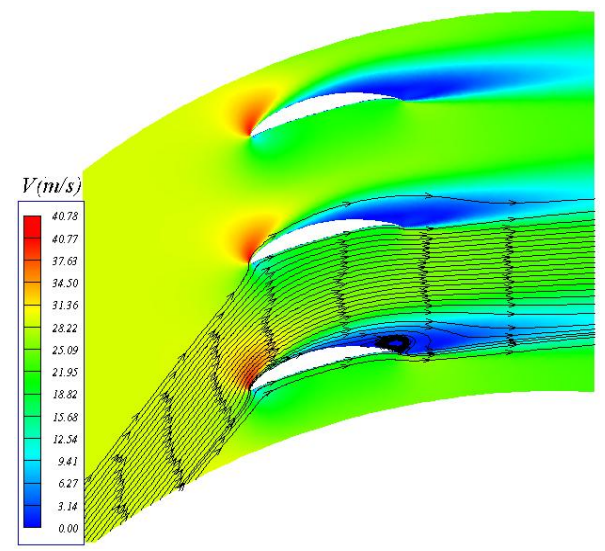

(a)

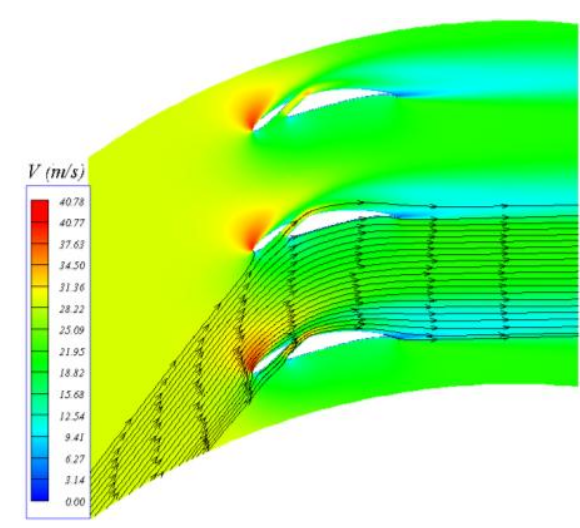

(b)

Fig. 15. Velocity contours and streamlines for the best configuration ( $\mathrm{X}=0.35 \mathrm{c}, \mathrm{Y}=7 \mathrm{~mm}$ and $\psi=30^{\circ}$ ), (a) Unslotted cascade, (b) Slotted cascade

\subsection{3-D Investigation with Optional Slotted Blading}

All results in this 3-D investigation are obtained with the same precedent operating conditions: inlet freestream Mach number $M=0.085$ and Reynolds number, based on blade chord, $R e=245000$. In the first part of this paragraph, numerical results for initial blade profile without slot are compared with slotted blade profile. Figure 16 shows the spanwise distribution of pitchwise averaged total loss coefficient for both cases calculated at an axial position $50 \%$ downstream from the trailing edge.

The total losses are strictly the sum of those due to the skin friction on blade surfaces and secondary losses. Since losses of the slotted case exhibit lower values over both regions end walls and mid span the slot has in this situation the capability to control secondary flow and boundary layer separation. 


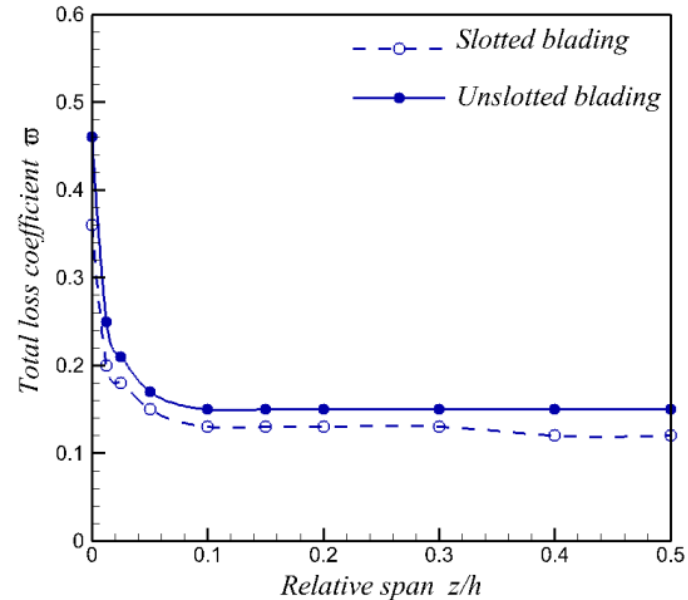

Fig. 16. Comparison of pitchwise mass averaged total pressure loss coefficient for initial blades (up) and slotted ones (bottom) at $\mathrm{x} / \mathrm{Cax}=1.5, \beta_{1}=52^{0}$

Figure 17 show the spanwise distribution of pitchwise averaged turning angle $\theta$. This turning angle distribution indicates that the slotted blade configuration gives better values as well. The secondary flow produces the areas of the overturning and under turning at around $12 \%$ span for each two cases with different level and evolution.

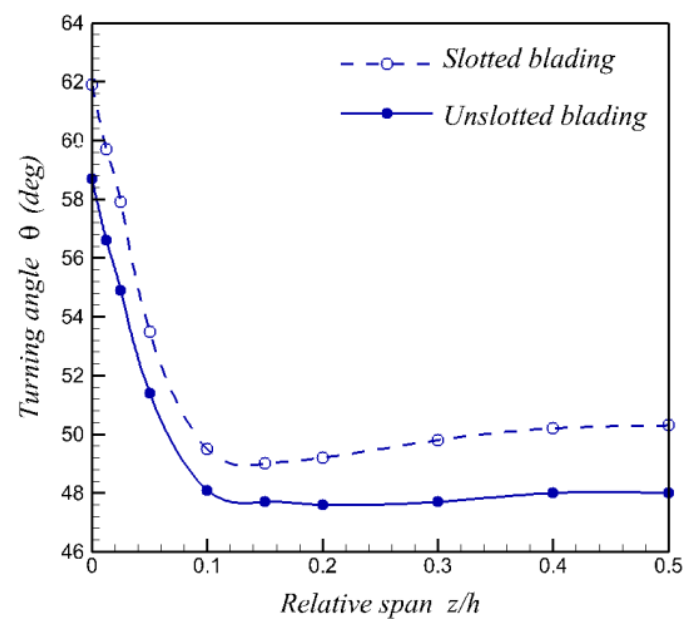

Fig. 17. Comparison of pitch wise mass averaged turning angle for initial blades (up) and slotted ones (bottom) at $\mathrm{x} / \mathrm{Cax}=1.5, \beta_{1}=52^{0}$

Figure 18(a) represent the wake identified by the contours plot of the total pressure loss coefficient on a cross plane located at $50 \%$ of axial chord downstream the blade trailing edge. It can be seen that the total pressure loss coefficient contours are symmetric about midspan in two cascades. In controlled case the loss coefficient is slightly skewed near the end wall and their highest values appear near the end wall under the effect of mixing with secondary flows.

The decreasing in total losses on the sidewall and blades surfaces downstream the slot is marked in the controlled cascade as depicted in Fig. 18(b). Therefore, the slot contributes to decrease the loss coefficient over the whole passage as it was already found in pure 2-D configuration.

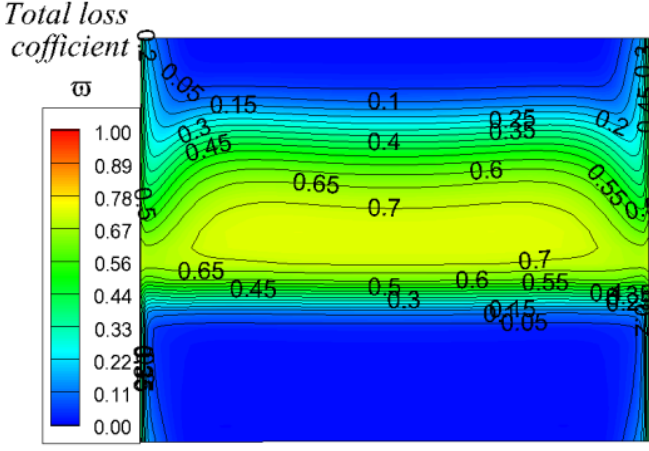

Total loss

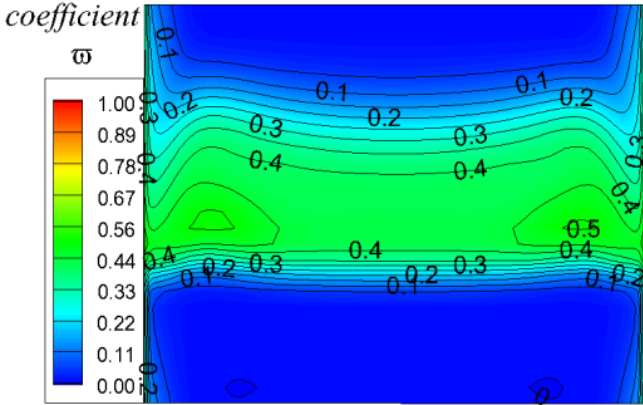

Fig. 18(a). Total pressure loss coefficient contours downstream the cascade for initial blades (up) and slotted ones (bottom), $\beta_{1}=52^{0}$ at $\mathrm{x} / \mathrm{Cax}=1.5$

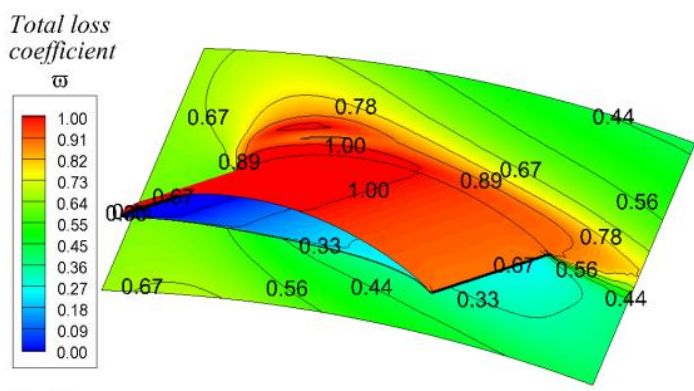

Total loss

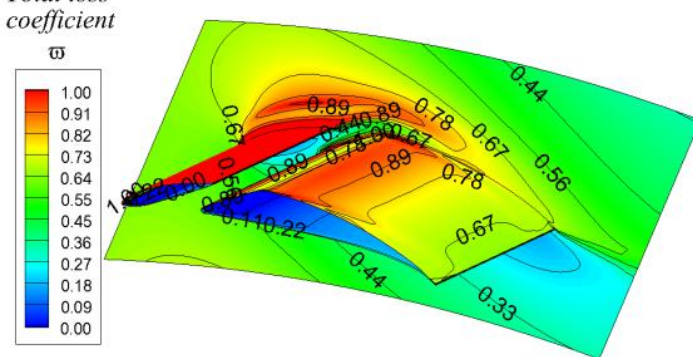

Fig. 18(b). Total pressure loss coefficient contours on blade and side wall for initial blades (up) and slotted ones (bottom), $\beta_{1}=52^{\circ}$

In order to give more details, Figs 19(a) and 19(b) represent the streamlines colored by axial velocity on blade and sidewall surfaces. The stall regions act as a blockage effect which reduces the main primary passage. The cascade without slot is influenced by a large amount of reversed flow in both corner and mid span which increase the blockage phenomenon in passage area. The end walls surfaces are qualified of stalled regions. The area of separation zones in the 
controlled blade changes significantly compared with the baseline cascade. The results for the off design condition is globally show a positive effects of the slot to mitigate the secondary losses.
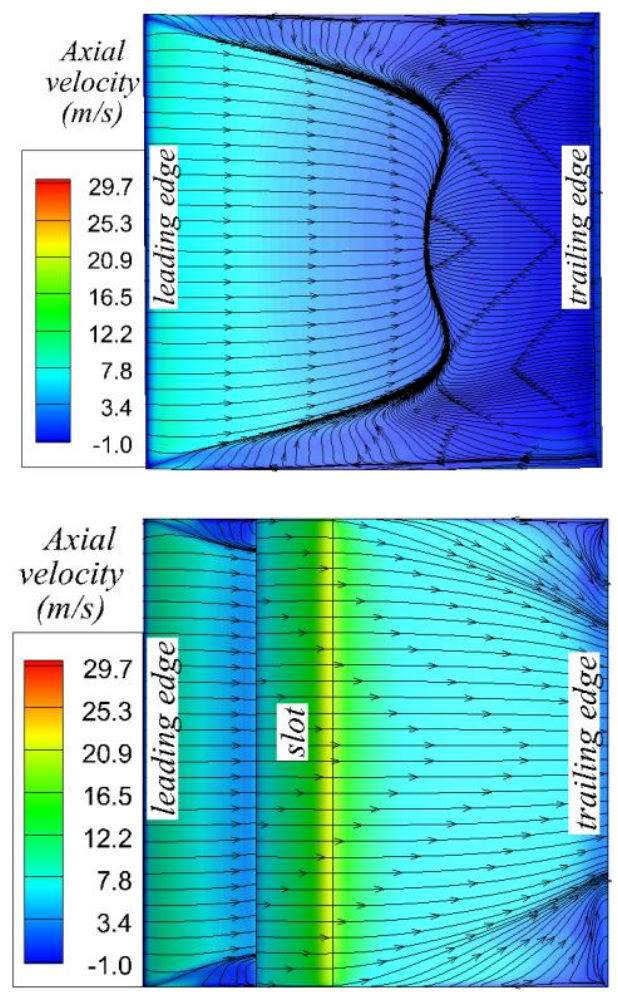

Fig. 19(a). Limiting streamlines on the suction surface for initial blades (up) and slotted ones (bottom), $\beta_{1}=52^{0}$
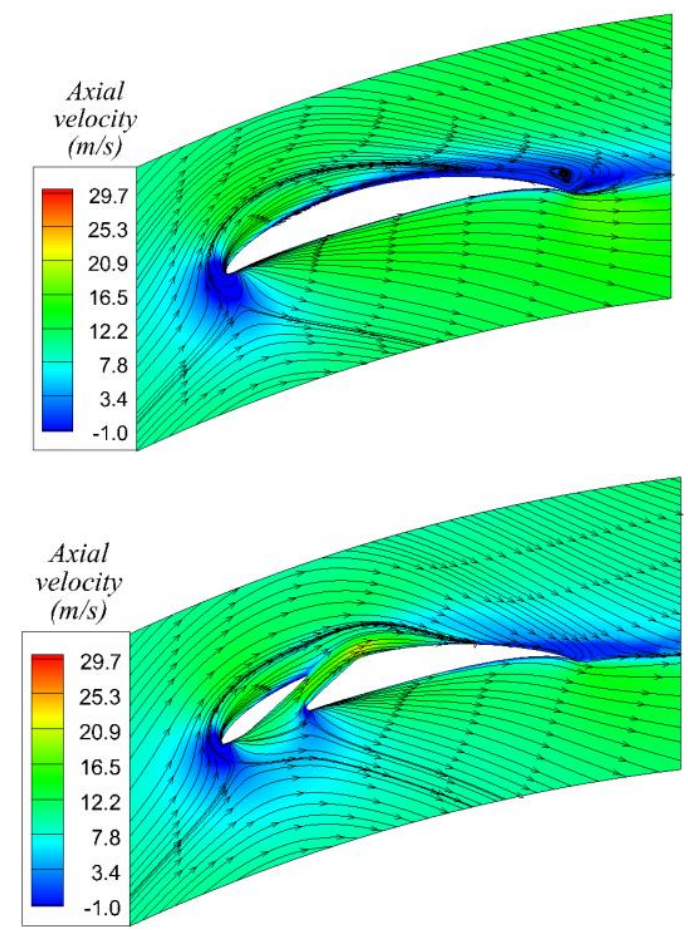

Fig. 19(b) Limiting streamlines on the endwall for initial blades (up) and slotted ones (bottom), $\beta_{1}=52^{0}$
It can be seen that the slot jet in the controlled cascade eliminate the boundary layer separations both in the corner on end wall and at mid span on the suction surface. However, the zone of suction surface inside the corner remains stalled.

The presence of the adverse pressure gradient in the blade passage and the cross flow from pressure to suction side in the boundary layer of the sidewall give a region characterized by the accumulation of low momentum fluid and the development of reverse flow. The structures of the vortices within the corner stall are illustrated in Figs. 20(a) and 20(b). In the cascade without slot the streamlines show a stronger reverse flow represented by a vortex perpendicular to the end wall surface and extended almost over the entire span.

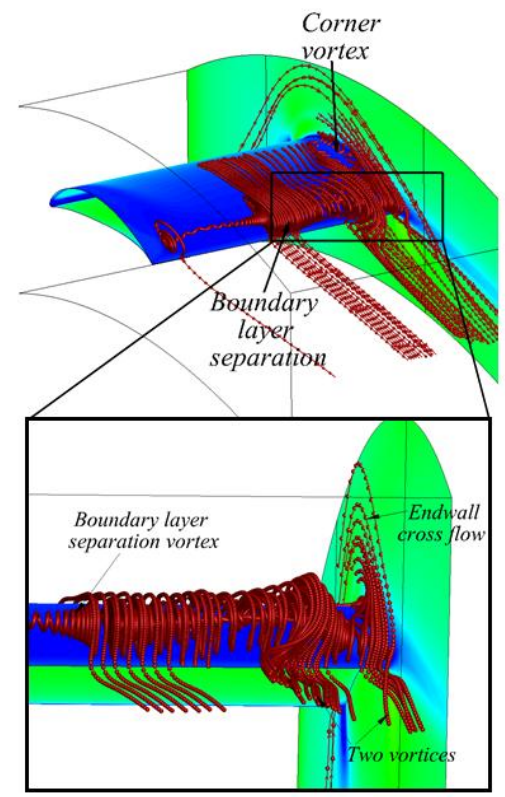

Fig. 20(a) Limiting streamlines on the blade and endwall for unslotted cascades, $\beta_{1}=52^{0}$

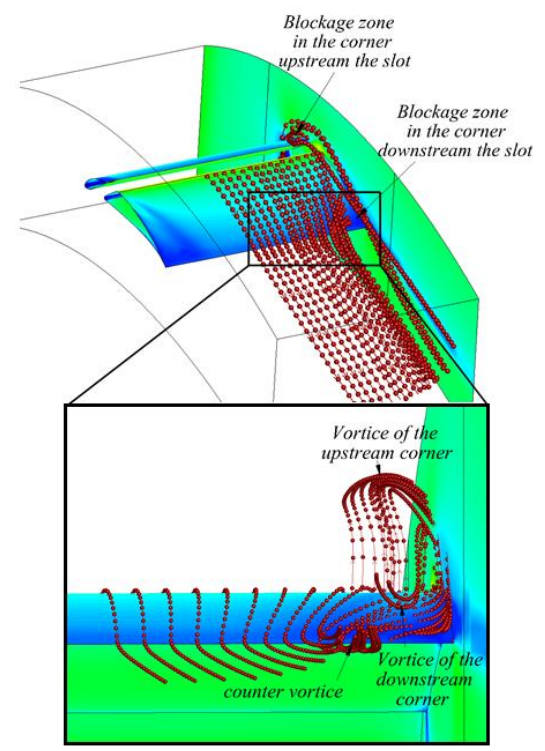

Fig. 20(b). Limiting streamlines on the blade and endwall for slotted cascades, $\beta_{1}=52^{0}$ 
The structures of the particle traces near the suction surface of the slotted blade are different from those obtained for initial case. In the former, the vortex due to the secondary flow is rolling up toward the mid span. This creates a well-known accumulation of low momentum fluid and the development of reverse flow. In the latter, the presence of slot creates two counter rotating vortices. These two vortices represent two legs of a single vortex and are situated near the trailing edge. The two counter vortices are the responsible for the obtaining of poor performances either total loss coefficient or turning angle.

This vortex is coexisted with another vortex due from the end wall flow. On the other hand, the three dimensional flow fields in corner stall of the controlled cascade indicate that the stronger counter vortex is weakened with the creation of another two vortexes. The onset of the first comes from the corner upstream the slot and the second is from the corner downstream the slot. Moreover, the vortex of boundary layer separation is eliminated. Seemingly, the control of profile vortex at stall condition has the predominant effect to decrease total loss and increase turning even three vortices may exist near end walls.

\section{CONClusion}

Numerical experimentations were performed in the highly loaded linear compressor cascade with NACA 65(18)10. The influences of location, width and slope of the slot were successively analyzed in twodimensional configuration. Under off design condition, the maximum relative reduction of loss coefficient was up to $28.3 \%$, when the slot jet was located approximately halfway between the minimum pressure point and the separation point, the slot width reached the threshold value and the slot slope became less stiff. Moreover, a difference of about $5^{0}$ between the turning angles with and without slot can be observed. In threedimensional situation, the optimal slot marks their ability to reduce the secondary flow structures and eliminate the boundary layer separation at midspan and the corner stall. This benefit appears in the case where the incoming flow angle was large and the boundary layer separation was occurred. Thus, another work under design condition might be proposed to explore the potential of slotted blading to control endwall flows. In the end, because the slot jet is exhausted with the same direction of the main flow, it has the capability to energize the boundary layer and control the separated flow on the suction surface of blade. However, as the secondary flows have a different structure and different direction the slot jet loses its impact to manipulate the secondary flow. Therefore, the solution to control the secondary flow structures is to use an active tool on the lateral endwalls. Like suction or blowing, these tools can be proposed in the future works in parallel with slotted bladings to improve aerodynamic performances of the axial compressors.

\section{REFERENCES}

Abdel-Fattah, A. (2012). Control of Separation Flow in a sudden enlargement. Journal of Applied Fluid Mechanics 5, 57-66.
Bruneau, C.H., E. Creusé, D. Depreyras, P. Giliéron and I. Mortazavi (2012). Active and Passive Flow Control around Simplified Ground Vehicules. Journal of Applied Fluid Mechanics 5, 89-93.

Emery, J. C., L.J. Herrig, J. R. Erwin and A.R.Felix (1958). Systematic Two-Dimensional Cascade of NACA 65-Series Compressor Blades at low Speeds. NASA report 1368.

Hergt, J., W. Klinner, C. Steinert, and E. Dorfner (2011). Detailed Flow Analysis of a Compressor Cascade with a Non-Axisymmetric Endwall Contour. $9^{\text {th }}$ European Conference on Turbomachinery, Turkey.

Linder, C.G. and B.A. Jones (1966). Single Stage Experimental Evaluation of Slotted Rotor and Stator Blading, Part I- Analysis and Design. NASA CR-54544, PWA FR-1713.

Linder, C.G. and B.A. Jones (1966). Single Stage Experimental Evaluation of Slotted Rotor and Stator Blading, Part III- Data and Performance for Slotted Rotor 1. NASA CR-54546, PWA FR-2110.

Linder, C.G. and B. A. Jones (1966). Single Stage Experimental Evaluation of Slotted Rotor and Stator Blading, Part VI- Data and Performance for Slotted Stator 1 and Flow Generation Rotor. NASA CR-54549, PWA FR-2286.

Mc.Glumphy, J., W. Ng, S.R. Wellborn and K. Severin, (2010). 3-D Numerical Investigation of Tandem Airfoils for a Core Compressor Rotor, ASME Journal of Turbomachinery 132, 1-9.

Mdouki, R., G. Bois, and A. Gahmousse (2011). Numerical Study of Passive Control with Slotted Blading in Highly Loaded Compressor Cascade at Low Mach number. International Journal of Fluid Machinery and Systems 4, 105-111.

Rockenbach, R. W., J. A. Brent, and B. A. Jones (1970). Single Stage Experimental Evaluation of Compressor Blading with Slots and Vortex Generators, Part I- Analysis and Design of Stages 4 and 5. NASA CR-72626, PWA FR-3461.

Rockenbach, R. W. and B. A. Jones (1970). Single Stage Experimental Evaluation of Compressor Blading with Slots and Wall Flow Fences. NASA CR-72635, PWA FR-3597.

Rockenbach, R. W. (1968). Single Stage Experimental Evaluation of Slotted Rotor and Stator Blading, Part IX-Final Report. CR-54553, PWA FR-2289.

Wennerstrom, C.G. (1990). Highly Loaded Axial Flow Compressors: History and Current Developments, ASME Journal of Turbomachinery 112, 567-578. 
M. Ramzi and G. AbdErrahmane / JAFM, Vol. 6, No. 4, pp. 571-580, 2013.

Yasuo, T., H. Hisashi, K. Yasuhiro, T. Minako and K. Yasushige (2005). Experimental and Numerical Investigations of Endwall Flow in a Bowed Compressor Cascade. AIAA 2005-3638, $41^{\text {st }}$ AIAA/ ASME/SAE/ASEE Joint Propulsion Conference and Exhibit, Arizona.
Zhou, M., R. Wang, Y. Bai, and L. Zeng (2008). Numerical research on effect of stator blade slot treatment on single stage compressor characteristic. Acta Aerodynamica Sinica, 26, 0258-25(2008)03-0400-05.

Zhou, M., R. Wang, Z. Cao and X. Zhang (2009). Effect of slot position and slot structure on performance of cascade, Acta Aerodynamica Sinica 27, 0258-1825(2009)01-0114-05. 